\title{
Physical activity promotion for patients transitioning to dialysis using the "Exercise is Medicine" framework: a multi-center randomized pragmatic trial (EIM-CKD trial) protocol
}

Ram Jagannathan', Susan Lynn Ziolkowski², Mary Beth Weber', Jason Cobb³ ${ }^{3}$ Nhat Pham ${ }^{4}$, Jin Long², Shuchi Anand ${ }^{2}$ and Felipe Lobelo ${ }^{1,5^{*}}$

\begin{abstract}
Background: Patients on dialysis are physically inactive, with most reporting activity levels below the fifth percentile of healthy age-matched groups. Several small studies have reported efficacy of diverse exercise interventions among persons with CKD and those on dialysis. However, no single intervention has been widely adopted in real-world practice, despite a clear need in this vulnerable population with high rates of mortality, frailty, and skilled nursing hospitalizations.

Methods/design: We describe a pragmatic clinical trial for an exercise intervention among patients transitioning to dialysis. We will use an existing framework - Exercise is Medicine (EIM) - developed by the American College of Sports Medicine. After undertaking formative qualitative research to tailor the EIM framework to the advanced CKD population (eGFR $<30 \mathrm{ml} / \mathrm{min} / 1.73 \mathrm{~m}^{2}$ ), we will randomize 96 patients from two regions -Atlanta and Bay Area-in two intervention arms with incremental levels of clinical-community integration: physical activity assessment during Nephrology clinical visit, brief counseling at pre-dialysis education, and physical activity wearable (group 1) versus group 1 intervention components plus a referral to a free, EIM practitioner-led group exercise program over 16 weeks (group 2; 8 week core intervention; 8-week follow up). We will assess efficacy by comparing between group differences in minutes/week of objectively measured moderate intensity physical activity. To evaluate implementation, we will use questionnaires for assessing barriers to referral, participation and retention along the path of the intervention. Further we will have a plan for dissemination of the intervention by partnering with relevant stakeholders.
\end{abstract}

Discussion: The overall goal is to inform the development of a practical, cost-conscious intervention "package" that addresses barriers and challenges to physical activity commonly faced by patients with advanced CKD and can be disseminated amongst interested practices.

Trial registration: ClinicalTrials.gov identifier (Dated:10/17/2017): NCT03311763.

Keywords: Chronic kidney disease, Exercise is medicine, Intervention, Physical activity, Qualitative interviews, Estimated glomerular filteration rate

\footnotetext{
* Correspondence: felipelobelo@emory.edu

${ }^{1}$ Hubert Department of Global Health, Rollins School of Public Health, Emory

University, 1518 Clifton Road NE, CNR 7051, Atlanta, GA 30322, USA

${ }^{5}$ Exercise is Medicine Global Research and Collaboration Center, 1518 Clifton

Road NE, CNR 7051, Atlanta, GA 30322, USA

Full list of author information is available at the end of the article
}

C The Author(s). 2018 Open Access This article is distributed under the terms of the Creative Commons Attribution 4.0 International License (http://creativecommons.org/licenses/by/4.0/), which permits unrestricted use, distribution, and reproduction in any medium, provided you give appropriate credit to the original author(s) and the source, provide a link to the Creative Commons license, and indicate if changes were made. The Creative Commons Public Domain Dedication waiver (http://creativecommons.org/publicdomain/zero/1.0/) applies to the data made available in this article, unless otherwise stated. 


\section{Background}

In 2017, 30 million adults or $15 \%$ of the United States population were estimated to have chronic kidney disease (CKD) [1]. It is well reported that minorities [2] have a higher reported incidence of CKD and end stage renal disease (ESRD), with African Americans and Hispanics having 3.9 and 1.5 (respectively) higher odds to develop ESRD compared to whites $[3,4]$. Despite medical advances, CKD remains a debilitating disease with a myriad of understudied symptoms and consequences, including insomnia [5], depression [6, 7], low muscle mass [8,9], and low physical activity (PA) levels [10-13], all contributing to substantial decrements in quality of life and adverse medical outcomes [7]. Nearly $95 \%$ of persons newly starting dialysis have physical fitness levels below the 20th percentile for the general population, and only $56.4 \%$ are reportedly able to walk one block [11]. Functional decline only accelerates after start of dialysis and leads to a negative spiral of deconditioning [14].

Prospective studies on patients with CKD requiring dialysis have shown a $60 \%$ or higher risk for mortality among those with the lowest level of PA compared to those with average or above average activity and/or fitness levels $[13,15,16]$. Further, sedentary behavior is associated with an increased risk for mortality among dialysis patients similar in magnitude to that of other well-established risk factors, such as a one-point reduction in serum albumin concentration [13]. Given the high cardiovascular mortality in persons with advanced CKD $[17,18]$ and the limited treatments available to patients who progress to end-stage kidney disease, there is a need to focus on promoting PA, a modifiable risk factor with potential for huge improvements in cardiovascular mortality, quality of life, and symptoms of uremia, which may improve tolerance dyalisis while countering the natural history of accelerated functional decline typically experienced by these patients [19]. The 12 month period preceding the anticipated start of dialysis may therefore be an important window of opportunity to implement PA interventions in this clinical population.

Previous intervention studies in persons with advanced CKD or those on dialysis have shown improvement in physical performance [20,21], health-related quality of life [21-23], and muscle strength or quality [20, 24, 25] with exercise therapy. Additional studies provide evidence for anti-inflammatory effects of exercise training among persons with advanced CKD [26]. The interventions described in these studies have differed in many aspects: setting (in dialysis center versus home based), intensity (prescribed versus patient driven), and type (aerobic, resistance, or combined) [27]. Despite these efforts, no single intervention design has emerged as a sustainable standard of care model for nephrology practices interested in implementing standardized PA programming as part of CKD management.
For the present study, we plan an exercise intervention that optimizes use of existing resources to integrate PA in the management of patients with advanced CKD transitioning to dialysis, with the main purpose of creating a transferrable intervention "package" that acknowledges and tackles implementation barriers in clinical and community settings. For this, we adapted the Exercise is Medicine (EIM) Framework, an initiative conceived in 2007 by the American College of Sports Medicine (ACSM) and colaunched by the American Medical Association, with the ultimate mission of implementing evidence-based PA intervention as standard of care for every patient [28]. This includes approaches for routinely integrating PA assessment, counseling and prescription/referral programs, particularly for patients with chronic diseases [29]. Herein, we describe a protocol to adapt the EIM framework for integrating PA promotion among patients transitioning to dialysis, integrating in-depth interviews with patients with CKD and feedback from community partners and directly test, in a pragmatic trial, the feasibility and short-term impact of this intervention on PA levels and secondary outcomes.

\section{Methods}

\section{Research rationale and objectives}

The overall goal of this research is to develop and validate a pragmatic and scalable intervention, working with patients and community partners to adopt the existing EIM framework to improve PA levels among patients with advanced CKD but not yet in dyalisis (Fig. 1). We have three main objectives:

(1) Develop a framework for a PA intervention, seeking input from patients using a qualitative methodology

(2) Synthesize and use the findings from Aim 1 to implement a PA intervention and test it in a randomized controlled pragmatic trial (8-week intervention and 8 week follo-up periods) in two settings enriched with minority populations.

(3) In addition to assessing short term effectiveness of the intervention, focus on implementation by assessing the barriers to referral, participation, and retention along the path of the planned intervention and on future scalability by engaging relevant stakeholders.

\section{Exercise is medicine framework}

The EIM Framework serves as the backbone for this study (www.exerciseismedicine.org) https://www.quantextual.co/ researcher/. This framework calls for routine assessment of PA during clinical interactions (called the "physical activity vital sign"), brief PA counseling, and a specific written PA prescription and/or referral to a community-based PA program, professional or resource. The PA prescription is often supplemented with referral to certified EIM practitioners (i.e. fitness professionals) that can lead exercise sessions, 


\begin{tabular}{|c|c|c|c|}
\hline \multicolumn{2}{|c|}{$\begin{array}{l}\text { Screening } \\
\text { Emory and Stanford University } \\
\text { Nephrology Service Clinical } \\
\text { Visit } \\
\text { (Anticipated } n=200 \text { ) }\end{array}$} & \multicolumn{2}{|c|}{$\begin{array}{l}\text { - Clinic staff pre-identifies patients with advanced CKD (eGFR }<30 \mathrm{ml} / \mathrm{min} / 1.73 \mathrm{~m}^{2} \text { ) } \\
\text { - } \\
\text { - }\end{array}$} \\
\hline \multicolumn{2}{|c|}{$\begin{array}{l}\text { Enrollment } \\
\text { Affiliated Dialysis Unit } \\
\text { Pre-dialysis Education Session } \\
(1 \text { monthly session at dialysis } \\
\text { unit) } \\
\text { (Anticipated } n=120)\end{array}$} & \multicolumn{2}{|c|}{$\begin{array}{ll}\text { - } & \text { Patients receive standard pre-dialysis education session } \\
\text { - } & \text { Referred or interested patients receive information about the study (or arranged for future } \\
\text { - } & \text { Those providing consent and confirmed eligible are enrolled } \\
\text { - Standard PA education and brief group-based lifestyle counseling session provided } \\
\text { - } & \text { Baseline assessments conducted (questionnaires; handgrip and 6-minute walk test) } \\
\end{array}$} \\
\hline \multicolumn{4}{|c|}{$\frac{\text { Randomization }}{\text { (Targeted } n=96)}$} \\
\hline & \multicolumn{2}{|c|}{ Group 1 Standard care advice $(n=48)$} & Group 2 Intervention $(n=48)$ \\
\hline$\frac{\frac{\text { Core }}{\text { Intervention }}}{16 \text { weeks }}$ & \multicolumn{2}{|c|}{$\begin{array}{ll} & \text { Initial contact with Exercise counseling } \\
\text { professional for personalized lifestyle change } \\
\text { plan (15 minutes via phone) } \\
\text { - Bi-weekly follow-up (via email, phone or SMS) } \\
\text { - } \quad \text { Patient engages in self-directed PA; weekly PA } \\
\text { synchronization via Garmin app } \\
\text { - Follow-up assessments conducted: } \\
\text { questionnaires; handgrip and 6-minute walk test } \\
\text { at } 12 \text { weeks }\end{array}$} & $\begin{array}{l}\text { - Referral to group-based PA program led by certified } \\
\text { fitness professionals (two sessions/week) } \\
\text { - Bi-weekly follow-up (via email, phone or SMS) } \\
\text { - Patient encouraged to further engage in self-directed } \\
\text { PA; Weekly PA synchronization via Garmin app } \\
\text { - Follow-up assessments conducted: questionnaires; } \\
\text { handgrip and 6-minute walk test at } 12 \text { weeks }\end{array}$ \\
\hline \multicolumn{4}{|c|}{$\begin{array}{l}\text { Fig. } 1 \text { Study design. Questionnaires: Demographics, age, sex, race, ethnicity, education, if employed/actively working, cause of CKD, vascular } \\
\text { access yes/no, other comorbidities, medications; Symptoms of Depression (CEDS-20), SF-12, Physical activity self-efficacy questionnaires; CKD } \\
\text { Chronic Kidney Disease, PA Physical activity' }\end{array}$} \\
\hline
\end{tabular}

often group-based, tailored to specific chronic diseases. A feedback mechanism from the EIM practitioner to both the patient and to the physician is also recommended, often using PA wearable monitoring technologies [30]. An evaluation framework for EIM implementation in health care settings has also been published [31].

\section{Research setting and team}

Participants for both the formative qualitative work and the intervention will be recruited through recommendation and referral from treating physicians at a Bay Area (Santa Clara Valley Medical Hospital Nephrology Clinic, an affiliate of Stanford University) and Atlanta (Emory Nephrology Clinic, Emory University). A group of seven nephrologists staff the Santa Clara Valley Medical Hospital Kidney clinic, serving a clinic population primarily insured under the county health plan and enriched with patients of Hispanic/Latino and Southeast Asian descent. Emory University's Midtown Kidney Clinic staffs seven academic nephrologists, and services a clinic population enriched with African American patients. In addition to the research teams and clinicians, fitness professionals certified in the Exercise is Medicine requirements https://www. quantextual.co/researcher/ will be recruited at each site to help lead PA counseling and group exercise sessions. The trial is prospectively registered on clinicaltrials.gov (NCT03311763) as of October 17, 2017.

\section{Participant recruitment}

The study design and inclusion/ exclusion criteria are shown in Table 1. English-speaking not yet on dialysis patients been seen at the two mentioned study clinical sites will complete a screening PA vital sign questionnaire, and thereafter study research coordinators will assess him or her for eligibility based on pre-specified inclusion/ exclusion criteria. Additionally, nurse recommendations and fliers briefly outlining the study posted within the kidney clinics will be used to help identify prospective participants. Potentially eligible patients will be contacted face-to-face or via telephone by a member of the research team to provide additional details on the study and its objectives. If ongoing interest is expressed, we will seek approval from nephrologists prior to enrolling. Research coordinators will obtain written consent from patients willing to participate in the trial. 
Table 1 Inclusion and exclusion criteria

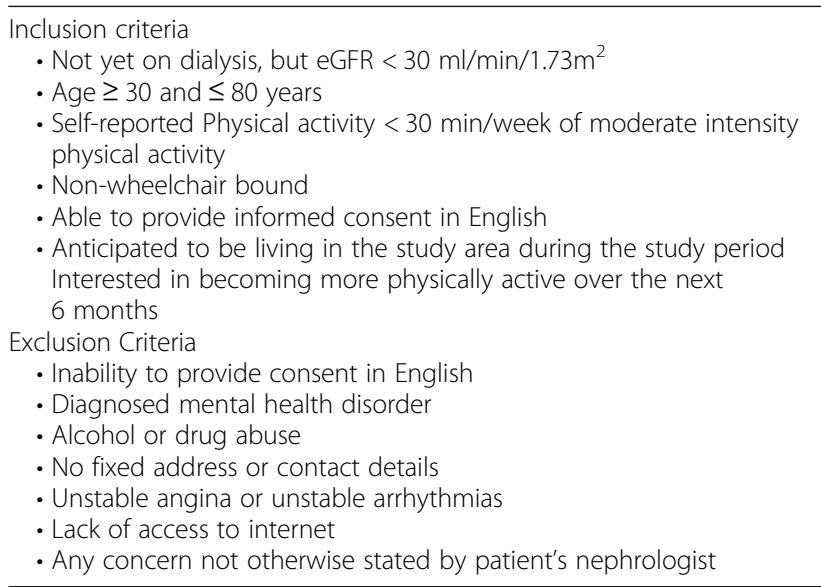

Formative data collection and intervention development For the tailoring of the EIM framework for patients with advanced CKD, we will use (1) feedback from community partners and (2) in-depth interviews with patients with CKD at the intervention site. We aim to interview a total of 20 participants (10 per site), stratified by sex (male/ female) and diabetes status (yes/NO) for a total of five patients in each sex-diabetes status strata. Participants will be asked to participate in a 1-h, semi-structured interview to discuss their exercise views and experiences, impact of CKD in exercise behaviors, opinions on planned intervention components (e.g., exercise classes, group-based exercise, mobile phone applications), and measures of programmatic success. Interviews will be digitally recorded using a smart phone equipped with specialized applications (Pocket Dictate for iPhone and Express Dictate for Andriod). Audiofiles will be transcribed verbatim for analysis. Transcripts will be reviewed and iterative processes will be employed to update the interview guide to add additional probes to gather more information or update questions if saturation is reached on a particular issue. An initial codebook including both deductive and inductive codes will be developed after reviewing all of the transcripts, and this codebook will be finalized through consultation with the study investigators. We will then conduct a thematic analysis, using MaxQDA (Verbi software) to assist with data manipulation and with structured comparisons of the data (men vs. women, individuals with and without diabetes, participants at each site) around key themes, to describe exercise behaviors and program recommendations from potential program users. The results of the analysis will then be applied to the EIM framework to finalize the intervention design.

\section{Baseline data collection}

Data collection will occur at baseline and at 8- (V1) and 16 weeks (V2) after the interventions (Table 2). Personal information, including relevant medical history will be collected prior to the initiation of testing or intervention upon receiving participant consent. This will include patient characteristics of age, sex, basic anthropometric measures (height, weight, BMI and waist circumference), relevant comorbidities and medications.

\section{Randomization}

Participants will be randomized to group 1 (low intensity) and group 2 (full -scale) intervention groups. Randomization will be conducted at a participant level prior to baseline testing by blocks of 16 via a computer-generated random number sequence by an independent researcher. Blinding of intervention group to clinical staff and participants will not be possible once the intervention starts.

\section{Trial interventions}

\section{Group 1 and 2}

\section{Brief physical activity counseling}

After study enrollment and assessment of the PA vital sign, a 30-min PA counseling session will be provided by the EIM - fitness professional. Evidence suggests that interventions should address individual, social and environmental factors to improve the likelihood of influencing behavior [32, 33]. Socio-ecological models of PA conceptualize and address the interaction and interdependency between individual, social and environmental factors [34, 35] and help identify the different environments where PA can be promoted, [36]. Health care and community settings are places where large proportions of the population can be reached and where PA can be effectively promoted [37-41]. By addressing multi-level influences for PA, the EIM intervention is rooted on socio-ecological approaches to increase the impact and sustainability of the intervention [33].

The PA counseling session will use brief-action planning, a highly structured, self-management support technique grounded in motivational Interviewing and behavior change strategies (goal setting, identify preferences and barriers, problem solving, stages of change and self-monitoring). Patients will be encouraged to incorporate moderate PA throughout the week to achieve 100-150 min/weekly, primarily through walking for leisure and transportation or other preferred activities [42].

\section{Werable physical activity monitor}

In order to support behavior change, all participants will receive a consumer-oriented "wearable" an associated smart-phone app to enable continued monitoring of $y$ sustained by study participants as part of the study's PA intervention programming (Fig. 2). The Garmin Vivofit 3 PA monitor was selected based on device usability and provision of evidence-based behavior change techniques, as summarized by a systematic content analysis of behavior change strategies implemented in commercially 
Table 2 Interventions examples

\begin{tabular}{|c|c|c|}
\hline Class format & Minutes & Teachable moments \\
\hline \multicolumn{3}{|l|}{ a. Strong bones and healthy hearts } \\
\hline Warm-up and rhythmic stretch & $10-12$ & $\begin{array}{l}\text { - Pre-Warm-Up: Perceived exertion, breathing and hydration. } \\
\text { - Action Planning: ROM safety through major/minor joints. } \\
\text { - Warm-up: Large to small muscles. }\end{array}$ \\
\hline Circuit Choreography & 15 & $\begin{array}{l}\text { - Selecting the right tubing resistance, benefits of strength work, benefits of cardio work } \\
\& \text { the plus for both in a circuit format. } \\
\text { - Resistance tubing safety. } \\
\text { - Breathing techniques for strength work. } \\
\text { - Cardio-cognitive benefits } \\
\text { - Benefits of tempo changes through half-time, at music tempo and double- time tempos. } \\
\text { - Benefits of non-dominant side movements. }\end{array}$ \\
\hline Cool-Down & 5 & - Benefits of lowering the heart rate progressively. \\
\hline Core Strengthening & 5 & - Benefits of concentric, eccentric strength training. \\
\hline Flexibility Training & $5-8$ & $\begin{array}{l}\text { - Option - Chair transfer technique from chair to floor and from floor to chair. } \\
\text { - Range of Motion versus Range of Movement. }\end{array}$ \\
\hline Relaxation \& Future Action Planning & $5-10$ & - Breaking the symptom cycle and self-managing pain, fatigue, frustration, etc. \\
\hline \multicolumn{3}{|l|}{ b. Stability \& Balance for Fall Prevention } \\
\hline Warm-up and rhythmic stretch & & $\begin{array}{l}\text { - Pre-Warm-Up: Perceived exertion, breathing and hydration. } \\
\text { - Action Planning: ROM safety through major/minor joints. } \\
\text { - Warm-up: Large to small muscles. }\end{array}$ \\
\hline Core stability and strength training & 5 & $\begin{array}{l}\text { - The muscle groups which compose the core. } \\
\text { - Benefits of breathing for increasing abdominal chest wall strength and flexibility }\end{array}$ \\
\hline Balance training & $5-10$ & $\begin{array}{l}\text { - Agility - changing center of gravity } \\
\text { - Lower body strength }\end{array}$ \\
\hline Stability training & $5-10$ & $\begin{array}{l}\text { - Stability in an unstable environment } \\
\text { - How to protect the lower back during extensions } \\
\text { - PNF stretching principles }\end{array}$ \\
\hline Flexibility & 10 & $\begin{array}{l}\text { - Stretch tight muscles for better posture } \\
\text { - Use breathing to deepen stretches }\end{array}$ \\
\hline Relaxation and Action planning & 10 & $\begin{array}{l}\text { - Breaking the symptom cycle to better self-manage pain, fatigue, fear, anger, frustration } \\
\text { and physical instabilities }\end{array}$ \\
\hline
\end{tabular}

available electronic activity monitors https://www.quan textual.co/researcher/, [43]. The Garmin Vivofit 3 measures and displays steps, calories, distance, minutes of activity intensity and sleep time. Furthermore, the wearable device links to a health IT HIPAA-certified platform (Quantextual Inc) https://www.quantextual.co/researcher/. that automatically syncs the user's PA information and allows them to self-monitor their progress, evaluate goals, interact with EIM study staff and share results with fitness professionals and providers on a free custom smartphone application (IOS or Android). This App will also enable the study staff to remotely monitor participants PA engagement. As such, the wearable device can be considered a component of the intervention in addition to providing process data.

\section{Group 2}

\section{Group-based physical activity program}

In addition to the intervention components previously listed, group 2 participants will also be referred to group-based PA program. We will procure an exercise space at each study site that is close to Nephrology Clinics and convenient for most participants to hold group-based exercise sessions. Before implementation, EIM fitness professionals will participate in a single 6-h training session conducted by one of the authors of the curriculum. The training consisted of an introduction and discussion of the curriculum objectives, demonstration of activities, and overview of the program's "Move and learn" activities.

At each location, we will make available blood pressure cuffs, glucometers and glucose strips for persons who experience symptoms related to hypotension or hypoglycemia during the class. Program sessions (8 persons per group) will be specifically designed for persons with CKD. Sessions will offer progressive aerobic conditioning, muscular strength and endurance, flexibility, and stability, balance and will be led by certified EIM fitness professionals [44].

Fitness professionals will follow the 2008 PA guidelines safety recommendations for PA programming tailored to persons with CKD and will be trained on communication skills and behavioral techniques (goal-setting, action 

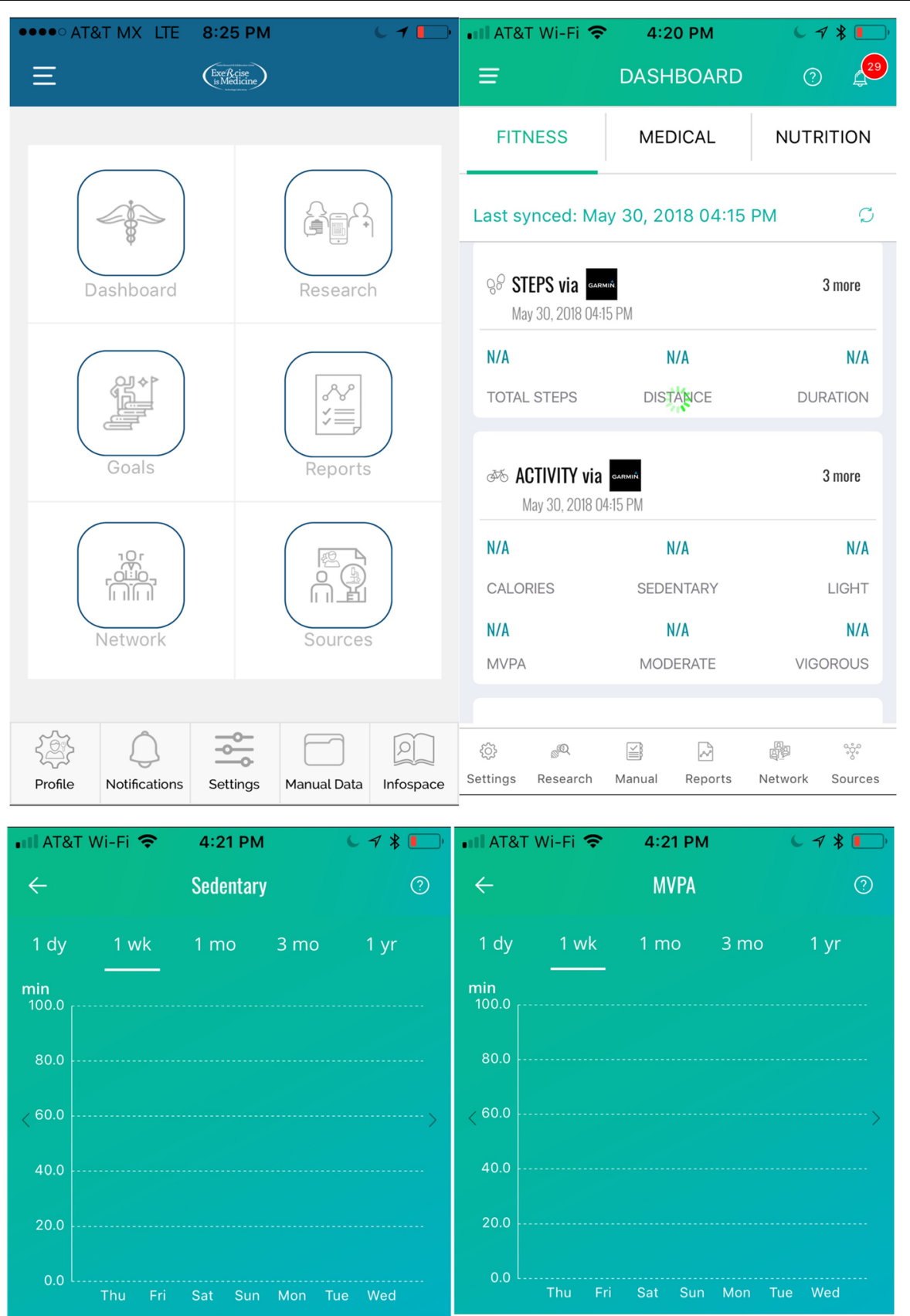

\begin{tabular}{|c|c|c|c|c|c|}
\hline Garmin $\nabla$ & & $\operatorname{ary}-$ & Garmin $\vee$ & & MVPA $\checkmark$ \\
\hline This week & & $\mathrm{N} / \mathrm{A}$ & This week & & $\mathrm{N} / \mathrm{A}$ \\
\hline Today & N/A & & Today & N/A & \\
\hline Yesterday & N/A & & Yesterday & $\mathrm{N} / \mathrm{A}$ & \\
\hline May 28, 2018 & $\mathrm{~N} / \mathrm{A}$ & & May 28, 2018 & $\mathrm{~N} / \mathrm{A}$ & \\
\hline
\end{tabular}

Fig. 2 Integration of Exercise is Medicine framework into Quantextual Smartphone Application 
planning, self-monitoring, provide instruction, feedback on performance, opportunities for social comparison, peer modeling, and social support) [45]. Two sessions/week will be offered, slowly progressing towards $50 \mathrm{~min}$ of lightto-moderate PA per session. Persons will also be encouraged to accumulate additional minutes of moderate PA throughout the week, primarily through increased walking for leisure and transportation. Interventions are structured around "Move and Learn" formats. They will include health education, led PA and lifestyle behavior change segments modeled after evidence-based sources (interventions examples are shown in Table 3) [46]. The social dynamics of the group will be used to forge strong, sustainable relationships with participants and reach appropriate minutes per week of PA, collect data using Health IT resources and provide guidance at the end of 8-week core intervention for transitioning to maintenance and self-managed PA. We will continue to offer the intervention even if the the participant initiates dialysis. For particiapnts who recently underwent surgery for dialysis access, we will encourage participation but limit arm exercise.

\section{Measurements}

Table 2 displays the data that we will collect during the study data. We plan to collect study data using Research Electronic Data Capture (REDCap) electronic data capture tools hosted at Emory University and Stanford University. REDCap is a secure, web-based application designed to support data capture for research studies providing 1) an intuitive interface for validated data entry; 2) audit trails for tracking data manipulation and export procedures; 3 ) automated export procedures for seamless data downloads to common statistical packages; and 4) procedures for importing data from external sources.

\section{Primary outcome \\ Minutes per week of physical activity}

The Study main outcome will be assessed objectively using the Garmin vivofit werable device. Weekly minutes of moderate-to-vigorous PA will be synchronized every week using the Quantextual application. In case of problems we will also manually sync the werable devices at the V1 and V2 measurement time points. In addition, weekly number of steps, sleep hours and minutes of sedentary time will also be collected.

\section{Secondary outomes \\ Questionnaires}

a) The Center for Epidemiological Studies-Depression questionnaire (CES-D): CES-D20, is a 20-item instrument that asks individuals to rate how often over the past week they experienced symptoms associated with depression, such as restless sleep, poor appetite, and feeling lonely. Response options range from 0 to 3 for each item $(0=$ rarely, 1 = little of the time, $2=$ much of the time, $3=$ almost all the time). Scores range from 0 to 60 , with high scores

Table 3 Study Procedures and measurements

\begin{tabular}{|c|c|c|c|}
\hline & Baseline & Follow-up: 1 & Follow-up: 2 \\
\hline & & 8 weeks & 16 weeks \\
\hline \multicolumn{4}{|l|}{ Self-reported } \\
\hline Physical activity vital sign & $\sqrt{ }$ & & \\
\hline Medical history (or any changes thereof) & $\sqrt{ }$ & $\sqrt{ }$ & $\sqrt{ }$ \\
\hline Quality of life (SF-12) & $\sqrt{ }$ & $\sqrt{ }$ & $\sqrt{ }$ \\
\hline Qualitative Interview $^{a}$ & $\sqrt{ }$ & & \\
\hline Adverse events & & $\sqrt{ }^{\mathrm{b}}$ & $\sqrt{ }$ \\
\hline \multicolumn{4}{|l|}{ MEASUREMENTS } \\
\hline Grip strength & $\sqrt{ }$ & $\sqrt{ }$ & $\sqrt{ }$ \\
\hline 6-min walk test & $\sqrt{ }$ & $\sqrt{ }$ & $\sqrt{ }$ \\
\hline Anthropometry (measured BMI, waist circumference) & $\sqrt{ }$ & $\sqrt{ }$ & $\sqrt{ }$ \\
\hline Blood pressure and blood glucose level & $\sqrt{ }$ & $\sqrt{ }$ & $\sqrt{ }$ \\
\hline Weekly physical activity as measured by Garmin PA monitor & $\sqrt{ }$ & $\sqrt{ }^{\mathrm{b}}$ & $\sqrt{ }$ \\
\hline \multicolumn{4}{|l|}{ INTERVENTIONS } \\
\hline Brief physical activity counseling & $\sqrt{ }$ & & \\
\hline Wearable physical activity monitor & $\sqrt{ }$ & $\sqrt{ }$ & $\sqrt{ }$ \\
\hline Group exercise sessions & & $\sqrt{ }$ & \\
\hline
\end{tabular}

${ }^{a}$ in a subset of patients recruited at start of study

bassessed continually during the study in G2 
indicating greater depressive symptoms. The CES-D also provides cutoff scores (e.g., 16 or greater) that aid in identifying individuals at risk for clinical depression, with good sensitivity and specificity and high internal consistency [47]

b) Short Form Health Survey (SF-12) [48]. The SF-12 questionnaire consists of 12 questions to measure functional health and wellbeing of the including a global question of perceived health status, to which the respondents indicate whether they think their overall health is excellent, very good, good, fair and poor. It covers the same eight health domains as the SF-36 with one or two questions per domain. The maximal number of points is 100 which in turn represents the best possible health condition and quality of life. SF-12 have been validated among dialysis patients and is in strong agreement with those derived from SF-36 in this population $(r=$ 0.94 for both physical and mental component) [49].

c) McAuley physical activity self-efficacy questionnaire [50]: The Exercise Self-Efficacy Scale assesses an individual's beliefs in their ability to continue exercising on a three time per week basis at moderate intensities for $40+$ minutes per session in the future. For each item, participants indicate their confidence to execute the behavior on a 100 -point percentage scale comprised of 10-point increments, ranging from $0 \%$ (not at all confident) to 100\% (highly confident). Total strength for each measure of self-efficacy is then calculated by summing the confidence ratings and dividing by the total number of items in the scale, resulting in a maximum possible efficacy score of 100.

\section{6-min walk test}

All eligible participants will undergo 6-min walk test according to American Thoracic Society's guideline [51]. In short, the test will be performed indoors on a $30 \mathrm{~m}$ straight course. Participants graded their dyspnea and fatigue perception on the 10-scale Borg scale. At the end of the 6-min period the distance, heart rate and blood pressure will be measured, and immediate fatigue and dyspnea scores will have reassessed. The participants will receive the same instructions before the walk and will be encouraged by the investigator who will repeat the set of phrases every 30 s during the walk.

\section{Grip strength}

We will carry out an assessment of grip strength in a standardized manner i.e., in neutral position of arm, forearm, and wrist. We will measure three consecutive attempts (with one-minute intervals in between) with a digital dynamometer (Takei 5401; Takei Scientific Instruments, Inc). All measurements will be done on setting II as suggested by Firrell and Crain and recorded in kilograms [52]. The same dynamometer will be employed for all participants. Calibration will be done automatically (electronic zero calibration system). For each participant, the arithmetic mean value of 3 measurements with the dynamometer will be used for statistical analysis. This method shows high repeatability [53].

\section{Statistical analysis}

\section{Sample size justification}

The study is powered on between-arm differences in the proportion achieving the primary outcome, minutes per week of moderate-level PA. Our sample size estimation is guided by PA counseling and referral review studies report an effect size between 0.09 and 0.71 for achieving PA guidelines [54-58]. We used the formula given in Vierron and Giraudeau [59] and Vittinghoff and Fitzmaurice [60] with an adjustment for the intraclass correlation coefficient (ICC), where $\mathrm{N}$ is the total sample size, the Z's are the critical value for type I $(\alpha=.05)$ and type II $(\beta=.2)$ error, $\sigma^{\wedge} 2$ is the variance in outcome, $\rho$ is the ICC, and $\pi$ is the proportion (50\%) of the $\mathrm{N}$ persons assigned to the treatment group. Based on the above formula, assuming an ICC of 0.5 [61], our total sample size of 98 will provide $89 \%$ power to detect a $100 \mathrm{~min} /$ week of moderate PA difference between groups, corresponding to an effect size of 0.46 . Considering an estimated $30 \%$ loss to long-term follow-up as reported in similar exercise intervention trials [62], our plan to enroll 98 participants should provide adequate power (80\%) to detect differences across intervention arms. Data gathered from those who withdraw from the study will not be used for statistical analyses. This has been accounted for when completing the power analysis, with an additional $20 \%$ added to the target sample size. Attrition may be for a number of reasons, which will be ascertained upon withdrawal where possible.

\section{Expected outcome}

Our primary outcomes is between-group difference in change in minutes/week of moderate-tovigorous PA between group 1 and 2 at 16 weeks. Based on our previous work, we expect greater improvement in grip strength, 6- min walk test, and PA among group 2 compared to group 1 participants. [54, 63]

\section{Quality assurance and trial coordination}

We will implement a clear set of quality assurance steps for trial coordination and delivery of the intervention. Pre-specified recruitment criteria and protocols, rigorous training of personnel at both sites, and monitoring of sites with feedback will help assure high-quality study findings. In order to deliver the multi-level PA intervention strategy uniformly across sites, we will conduct training and certification, provide standardized tools, conduct site-initiation visits, team meetings, and study 
staff role into each existing patient flow. Data will be collected using standardized tools and instruments. All safety concerns, acute events [e.g., myocardial infarction injuries], or mortality noted during 16-weeks of trial follow-up will be compared between study arms and reported to oversight committees.

\section{Data analysis}

Principal Investigators (Stanford University and Emory University) will be given access to the cleaned data sets. Project data sets will be housed on the REDCap hosted by the respective universities and all data sets will be password protected. To ensure confidentiality, data dispersed to project team members will be blinded of any identifying participant information.

Between-group differences in primary and secondary outcomes will be assessed at 8 and 16 weeks. We will test for differences in primary and secondary outcomes to assess treatment effects using multivariate regression models adjusting for baseline characteristics. We will use mixed-effects linear and logistic regression models to predict changes in continuous PA levels and probability of achieving PA recommendations by regressing study arm, study centers, and an interaction between study arm*centers. The mixed model will include one random effect term accounting for the correlation within study center. Analysis of effectiveness will be according to an intention-to-treat analysis including all enrolled and randomized persons into our analysis. Irrespective of compliance with treatment, all participants will be encouraged to continue with the scheduled outcome evaluation until the end of the study. However, as high as up to $30 \%$ loss to long-term follow-up would be expected. The characteristics for those participants without complete follow-up will be examined. If missing data is determined to be random, we will perform a sensitivity analysis using multiple imputation-based methods. If missing observations cannot be assumed to be random, pattern mixture models will be considered [64].

\section{Potential risks}

Adverse effects of exercise: Minor risks include muscle soreness, light-headedness/dizziness, and weakness. Physical activity may lead to more serious health problems such as a heart attack and sudden death. However, this is very rare. Estimates of sudden cardiac death range from 0 to 2 per 100,000 h of physical activity. Physical Activity may also lead to acute spikes in blood pressure and/or blood glucose if the patient is not well-controlled. Group exercise classes will be led by trained professionals who are able to offer suggestions to limit and prevent these adverse events. Session will also include measurements of blood pressure and glucose to identify potential contra-indications for exercise that day. Fitness professionals will be trained to recognize medical emergencies and seek emergency care if needed. A potential participant's nephrologist will be informed of their patient's possible participation in our trial and if they determine a patient is medically unfit to participate, they will be excluded from the study.

Participants will be informed at each visit and exercise session that if at any point during the study they develop chest pain, shortness of breath, lightheadedness, or fall they should stop activity immediately and inform study staff if currently at a study visit or call 911 if at home. In the event of milder symptoms at home, they are advised to call study coordinators to inform them of their symptoms as they may alter future intervention. Participants' nephrologist will be notified of all events and reported symptoms and will review the case before study should be continues.

\section{Loss of confidentiality}

Participation in the study will mean that some protected health information will need to be obtained from study participants. This information will only be shared with persons involved in the study and listed on the informed consent form. All information will be de-identified if used for other purposes such as medical publications.

\section{Trial status}

Study is currently ongoing and recruitment has begun at Stanford University site. Inclusion is expected to be complete by early 2019 .

\section{Discussion}

Physical inactivity is a major problem in persons with advanced CKD and transitioning to dialysis and a modifiable risk factor for many important outcomes including function, quality of life, and mortality. Several attempts have been made to make exercise more convenient for CKD patients by different intervention modalities such as intradialytic exercise programs using cycle ergometers [21] and various resistance exercise programs [65]. Despite these attempts at tailored interventions, patients are not adhering to exercise regimens. When Konstantinidou et al. [66] compared intradialytic exercise, home based exercise, and rehabilitation center-based exercise, the group that participated in the intradialytic exercise program had a dropout rate of $16.7 \%$, as compared to $23.8 \%$ dropout rate for patients in the rehabilitation center-based group and $16.7 \%$ in the home-based exercise group.

In order for clinicians and health care systems to create tailored exercise interventions that promote exercise adherence, further examination of barriers to and motivators for exercise in CKD patients is needed. Our interdisciplinary approach includes vital input from the target populations, fosters physician-patient interactions around 
this topic, and is based on use of existing clinical and community resources, supplemented with trained EIM fitness professionals for whom we will develop a curriculum specific to persons with advanced kidney disease. If shown to be efficacious and effectively implemented, our research will be translatable immediately within the two major practices involved in the study and with modest effort, to other practices within the region.

Our previous "proof of principle" feasibility study which compared the effectiveness of the supplemented EIM protocol (PA vital sign + exercise referral arm) compared to the EIM protocol alone (PA vital sign) in the general population demonstrated a greater improvement in PA among individuals in the referal group (changes in self-reported PA: rerefal group: $+250 \mathrm{~min} /$ week vs.

EIM arm: $-38.6 \mathrm{~min} /$ week; $p=0.0002$ ) [67]. Our group also evaluated the effectiveness of a PA referral program analogous to the EIM framework for hypertensive adults in Mexico's Social Security healthcare system and found this strategy was well accepted by practitioners and particiapnts, with significant improvements in moderate-to-vigorous PA minutes reductions in diastolic blood pressure $(4.5 \mathrm{mmHg})$, waist circumference $(2.5 \mathrm{~cm})$ and fasting blood glucose (5 mg/dl) all $p<0.05$ in the referral group compared to usual care $[63,68]$. Therefore, the EIM framework has a track record for feasibility of implementation in clinical settings with evident gains in PA among participants especially when referal to group-based exercise sessions led by certified fitness professionals We believe this pragmatic approach has great potential to engage nephrologists and patients with advanced CKD in positive, motivational interactions with specific, well-designed follow through plans.

This study will be the first to assess the EIM framework and additional exercise session referral strategy in the CKD population. This will allow for potential application of this protocol in other nephrology practices with little clinical burden on existing staff. A further strength of our study is the adaptation of this program in two racially and ethnically diverse areas. In addition, the intervention is built on proven science and tailored to the unique needs of the study population as assessed in a series of formative, in-depth interviews. The limitations of this study include potential selection bias of highly motivated persons within nephrology practices, so effects may not be generalizable to the entire CKD population. Further, due to the short duration of the study, long-term outcomes such as mortality or delays in start of dialysis cannot be obtained with sufficient power.

Once complete, we hope to adapt the main intervention according to the successes and challenges of the initial trial. We will contact dialysis organizations (large or small) and seek individual meetings with administrators at dialysis and insurance (i.e Medicare, private) organizations to create a larger roll out. Working with the ACSM we can easily disseminate the personnel training and equipment used in the intervention as a package for larger implementation in the US and internationally as part of the Global EIM initiative.

\section{Conclusion}

In sum, the current feasibility trial will be an important step towards an important and urgently-needed intervention for patients on dialysis who are extremely sedentary. We will employ the strengths of the health systems and clinicians to initiate PA promotion and referrals to community programming. We will supplement existing community resources by providing trained and "linked-in" EIM practitioners with specific knowledge of the challenges facing patients transitioning to dialysis. We plan to inform the development of a pragmatic, cost-conscious and scalable intervention 'package' that facilitates integration of PA into the routine care of CKD clinical populations.

\section{Abbreviations}

ACSM: American College of Sports Medicine; CES-D: Center for Epidemiological Studies-Depression questionnaire; CKD: Chronic Kidney Diseases; EIM: Exercise is Medicine; PA: Physical activity; SF12: Short Form Health Survey

\section{Acknowledgements}

The authors acknowledge the assistance of Kanya Rajagopalan and Jacqueline Aiko from Emory University school of Public Health for their support in intervention components and manual of procedures respectively.

\section{Funding}

Our study has been funded by a grant for pragmatic clinical research by the Normon S Coplon Satellite Grant Committee. Satellite Healthcare is a nonprofit hemodialysis provider.

\section{Avilability of data and materials}

The datasets used and/or analyzed during the current study will be available from the principal investigator $(F L, S A)$ on reasonable request.

\section{Authors' contributions}

$J R$ is the research coordinator, SLZ, JC, NP, SA and FL are investigators/ subinvestigators in the respective study centers, MBW consult for focus group interview and $J \mathrm{~L}$ is the statistical advisor. JR drafted the manuscript, and all co-authors reviewed and revised the article. All authors read and approved the final manuscript.

\section{Ethics approval and consent to participate}

The trial has been approved by the institutional review boards of Stanford (IRB Registration \#: 43198) and Emory University (IRB\#: IRB00099894). Oral and written informed consent to participation will be obtained from all study participants by study personnel prior to any study related procedure. All participants receive both written and oral information about the study before consenting. Any modifications to the protocol which may impact on the conduct of the study, including changes of study objectives, study design, patient population, sample sizes, study procedures, or significant administrative aspects will require a formal amendment to the protocol and approved by the ethical review board of Stanford and Emory University before its commencement.

Consent for publication

Not applicable.

Competing interests

The authors declare that they have no competing interests. 


\section{Publisher's Note}

Springer Nature remains neutral with regard to jurisdictional claims in published maps and institutional affiliations.

\section{Author details ${ }^{3}$ Renal Division, Department of Medicine, Emory University School of USA. \\ Received: 20 June 2018 Accepted: 2 September 2018 Published online: 12 September 2018}

'Hubert Department of Global Health, Rollins School of Public Health, Emory University, 1518 Clifton Road NE, CNR 7051, Atlanta, GA 30322, USA. 'Division of Nephrology, Stanford University School of Medicine, Palo Alto, CA, USA. Medicine, Atlanta, Georgia, USA. ${ }^{4}$ Division of Nephrology, Santa Clara Valley Medical Center, San Jose, CA, USA. ${ }^{5}$ Exercise is Medicine Global Research and Collaboration Center, 1518 Clifton Road NE, CNR 7051, Atlanta, GA 30322,

\section{References}

1. Centers for Disease Control and Prevention. Chronic kidney disease surveillance system website. http://www.cdc.gov/ckd. Accessed 6 Apr 2018.

2. Powe NR, Melamed ML. Racial disparities in the optimal delivery of chronic kidney disease care. Med Clin N Am. 2005;89(3):475-88.

3. Norris K, Nissenson AR. Race, gender, and socioeconomic disparities in CKD in the United States. J Am Soc Nephrol. 2008;19(7):1261-70.

4. Xue JL, Ma JZ, Louis TA, Collins AJ. Forecast of the number of patients with end-stage renal disease in the United States to the year 2010. J Am Soc Nephrol. 2001;12(12):2753-8.

5. Nigam G, Camacho M, Chang ET, Riaz M. Exploring sleep disorders in patients with chronic kidney disease. Nat Sci Sleep. 2018;10:35-43.

6. Palmer S, Vecchio M, Craig JC, Tonelli M, Johnson DW, Nicolucci A, Pellegrini F, Saglimbene V, Logroscino G, Fishbane S, et al. Prevalence of depression in chronic kidney disease: systematic review and meta-analysis of observational studies. Kidney Int. 2013;84(1):179-91.

7. Abdel-Kader K, Unruh ML, Weisbord SD. Symptom burden, depression, and quality of life in chronic and end-stage kidney disease. Clin J Am Soc Nephrol. 2009;4(6):1057-64

8. Isoyama N, Qureshi AR, Avesani CM, Lindholm B, Barany P, Heimburger O, Cederholm T, Stenvinkel P, Carrero JJ. Comparative associations of muscle mass and muscle strength with mortality in dialysis patients. Clin J Am Soc Nephrol. 2014;9(10):1720-8

9. Stenvinkel P, Carrero JJ, von Walden F, Ikizler TA, Nader GA. Muscle wasting in end-stage renal disease promulgates premature death: established, emerging and potential novel treatment strategies. Nephrol Dial Transplant. 2016;31(7):1070-7

10. Johansen KL, Chertow GM, Ng AV, Mulligan K, Carey S, Schoenfeld PY, KentBraun JA. Physical activity levels in patients on hemodialysis and healthy sedentary controls. Kidney Int. 2000;57(6):2564-70.

11. Johansen KL, Chertow GM, Kutner NG, Dalrymple LS, Grimes BA, Kaysen GA. Low level of self-reported physical activity in ambulatory patients new to dialysis. Kidney Int. 2010;78(11):1164-70.

12. Stack AG, Molony DA, Rives T, Tyson J, Murthy BV. Association of physical activity with mortality in the US dialysis population. Am J Kidney Dis. 2005; 45(4):690-701

13. O'Hare AM, Tawney K, Bacchetti P, Johansen KL. Decreased survival among sedentary patients undergoing dialysis: results from the dialysis morbidity and mortality study wave 2. Am J Kidney Dis. 2003:41(2):447-54.

14. Kurella Tamura M, Covinsky KE, Chertow GM, Yaffe K, Landefeld CS, McCulloch CE. Functional status of elderly adults before and after initiation of dialysis. N Engl J Med. 2009;361(16):1539-47.

15. Johansen KL, Kaysen GA, Dalrymple LS, Grimes BA, Glidden DV, Anand S, Chertow GM. Association of physical activity with survival among ambulatory patients on dialysis: the comprehensive Dialysis study. Clin J Am Soc Nephrol. 2013:8(2):248-53.

16. Lopes AA, Lantz B, Morgenstern H, Wang M, Bieber BA, Gillespie BW, Li Y, Painter $\mathrm{P}$, Jacobson SH, Rayner HC, et al. Associations of self-reported physical activity types and levels with quality of life, depression symptoms, and mortality in hemodialysis patients: the DOPPS. Clin J Am Soc Nephrol. 2014;9(10):1702-12.

17. Collins AJ. Cardiovascular mortality in end-stage renal disease. Am J Med Sci. 2003;325(4):163-7.
18. Tonelli M, Wiebe N, Culleton B, House A, Rabbat C, Fok M, McAlister F, Garg AX. Chronic kidney disease and mortality risk: a systematic review. J Am Soc Nephrol. 2006;17(7):2034-47.

19. Barcellos FC, Santos IS, Umpierre D, Bohlke M, Hallal PC. Effects of exercise in the whole spectrum of chronic kidney disease: a systematic review. Clin Kidney J. 2015:8(6):753-65.

20. Johansen KL, Painter PL, Sakkas GK, Gordon P, Doyle J, Shubert T. Effects of resistance exercise training and nandrolone decanoate on body composition and muscle function among patients who receive hemodialysis: a randomized controlled trial. J Am Soc Nephrol. 2006;17(8):2307-14.

21. Painter P, Carlson L, Carey S, Paul SM, Myll J. Physical functioning and health-related quality-of-life changes with exercise training in hemodialysis patients. Am J Kidney Dis. 2000;35(3):482-92.

22. Kouidi E, Grekas D, Deligiannis A, Tourkantonis A. Outcomes of long-term exercise training in dialysis patients: comparison of two training programs. Clin Nephrol. 2004;61(Suppl 1):S31-8.

23. Tawney KW, Tawney PJ, Hladik G, Hogan SL, Falk RJ, Weaver C, Moore DT, Lee MY. The life readiness program: a physical rehabilitation program for patients on hemodialysis. Am J Kidney Dis. 2000:36(3):581-91.

24. Cheema B, Abas H, Smith B, O'Sullivan A, Chan M, Patwardhan A, Kelly J, Gillin A, Pang G, Lloyd B, et al. Progressive exercise for anabolism in kidney disease (PEAK): a randomized, controlled trial of resistance training during hemodialysis. J Am Soc Nephrol. 2007;18(5):1594-601.

25. Heiwe S, Tollback A, Clyne N. Twelve weeks of exercise training increases muscle function and walking capacity in elderly predialysis patients and healthy subjects. Nephron. 2001;88(1):48-56.

26. Viana JL, Kosmadakis GC, Watson EL, Bevington A, Feehally J, Bishop NC, Smith AC. Evidence for anti-inflammatory effects of exercise in CKD. J Am Soc Nephrol. 2014;25(9):2121-30

27. Heiwe $\mathrm{S}$, Jacobson $\mathrm{SH}$. Exercise training for adults with chronic kidney disease. Cochrane Database Syst Rev. 2011;10:CD003236.

28. Lobelo F, Stoutenberg M, Hutber A. The exercise is medicine Global Health initiative: a 2014 update. Br J Sports Med. 2014;48(22):1627-33.

29. Lobelo F, Rohm Young D, Sallis R, Garber MD, Billinger SA, Duperly J, Hutber A, Pate RR, Thomas RJ, Widlansky ME, et al. Routine assessment and promotion of physical activity in healthcare settings: a scientific statement from the American Heart Association. Circulation. 2018;137(18):e495-522.

30. Lobelo F, Kelli HM, Tejedor SC, Pratt M, McConnell MV, Martin SS, Welk GJ. The wild wild west: a framework to integrate mHealth software applications and wearables to support physical activity assessment, counseling and interventions for cardiovascular disease risk reduction. Prog Cardiovasc Dis. 2016;58(6):584-94.

31. Stoutenberg M, Galaviz KI, Lobelo F, Joy E, Heath GW, Hutber A, Estabrooks P. A pragmatic application of the RE-AIM framework for evaluating the implementation of physical activity as a standard of care in health systems. Prev Chronic Dis, 2018:15:E54.

32. Bauman AE, Reis RS, Sallis JF, Wells JC, Loos JFR, Martin BW. Correlates of physical activity: why are some people physically active and others not? Lancet. 2012;380:258-71.

33. Haggis C, Sims-Gould J, Winters M, Gutteridge K, McKay HA. Sustained impact of community-based physical activity interventions: key elements for success. BMC Public Health. 2013:13:892.

34. Sallis J, Bauman A, Pratt M. Environmental and policy interventions to promote physical activity. Am J Prev Med. 1998;15(4):379-97.

35. Spence JC, Lee RE. Toward a comprehensive model of physical activity. Psychol Sport Exerc. 2003:4(1):7-24

36. Sallis JF, Glanz K. Physical activity and food environments: solutions to the obesity epidemic. The Milbank Quarterly. 2009;87(1):123-54.

37. Patrick K, Pratt M, Sallis RE. The healthcare Sector's role in the U.S. National Physical Activity Plan. J Phys Act Health. 2009;6(2):S211-9.

38. Meyer P, Kayser B, Kossovsky MP, Sigaud P, Carballo D, Keller PF, Martin XE, Farpour-Lambert N, Pichard C, Mach F. Stairs instead of elevators at workplace: cardioprotective effects of a pragmatic intervention. Eur J Cardiovasc Prev Rehabil. 2010;17(5):569-75.

39. Orrow G, Kinmonth A-L, Sanderson S, Sutton S. Effectiveness of physical activity promotion based in primary care: systematic review and meta-analysis of randomised controlled trials. BMJ (Clinical research ed). 2012;344:-e1389.

40. Task Force on Community Preventive Services. Recommendations to increase physical activity in communities. Am J Prev Med. 2002:22(4S):67-72.

41. Tulloch H, Fortier M, Hogg W. Physical activity counseling in primary care: who has and who should be counseling? Patient Educ Couns. 2006:64(1-3): $6-20$ 
42. Gutnick D, Reims K, Davis C, Gainforth H, Jay M, Cole S: Brief Action Planning to Facilitate Behavior Change and Support Patient SelfManagement. J Clin Outcomes Manage 2014, 21(1).

43. Sanders JP, Loveday A, Pearson N, Edwardson C, Yates T, Biddle SJ, Esliger DW. Devices for self-monitoring sedentary time or physical activity: a scoping review. J Med Internet Res. 2016;18(5):e90.

44. Exercise is Medicine Credential. https://www.acsm.org/get-stay-certified/getcertified/specialization/eim-credential. Accessed 6 Sept 2018.

45. Dixon $\mathrm{D}$, Johnston M. Health behaviours change competency framework: competencies to deliver interventions to change lifestyle behaviours that affect health. In. The Scottish Government: Edinburgh; 2010.

46. Diabetes Prevention Program Research Group. 10-year follow-up of diabetes incidence and weight loss in the diabetes prevention program outcomes study. Lancet. 2009;374(9702):1677-86.

47. Carleton RN, Thibodeau MA, Teale MJ, Welch PG, Abrams MP, Robinson T, Asmundson GJ. The center for epidemiologic studies depression scale: a review with a theoretical and empirical examination of item content and factor structure. PLoS One. 2013;8(3):e58067.

48. Ware J Jr, Kosinski M, Keller SD. A 12-item short-form health survey: construction of scales and preliminary tests of reliability and validity. Med Care. 1996;34(3):220-33.

49. Lacson E Jr, Xu J, Lin SF, Dean SG, Lazarus JM, Hakim RM. A comparison of SF-36 and SF-12 composite scores and subsequent hospitalization and mortality risks in long-term dialysis patients. Clin J Am Soc Nephrol. 2010;5(2):252-60.

50. McAuley E. Self-efficacy and the maintenance of exercise participation in older adults. J Behav Med. 1993;16(1):103-13.

51. Laboratories ATSCoPSFCPF. ATS statement: guidelines for the six-minute walk test. Am J Respir Crit Care Med. 2002;166(1):111-7.

52. Firrell JC, Crain GM. Which setting of the dynamometer provides maximal grip strength? J Hand Surg Am. 1996;21(3):397-401.

53. Haider S, Luger E, Kapan A, Titze S, Lackinger C, Schindler KE, Dorner TE. Associations between daily physical activity, handgrip strength, muscle mass, physical performance and quality of life in prefrail and frail community-dwelling older adults. Qual Life Res. 2016;25(12):3129-38.

54. Lobelo F, de Quevedo IG. The Evidence in Support of Physicians and Health Care Providers as Physical Activity Role Models. Am J Lifestyle Med. 2016;10: 36-52.

55. Vuori IM, Lavie CJ, Blair SN. Physical activity promotion in the health care system. Mayo Clin Proc. 2013:88(12):1446-61.

56. Vuori IM, Lavie CJ, Blair SN. Physical Activity Promotion in the Health Care System. Mayo Clin Proc. 2013;88:1446-61.

57. Lin JS, O'Connor E, Whitlock EP, Beil TL, Zuber SP, Perdue LA, Plaut D, Lutz K. Behavioral Counseling to Promote Physical Activity and a Healthful Diet to Prevent Cardiovascular Disease in Adults: Update of the Evidence for the U.S. Preventive Services Task Force. Evidence Synthesis No. 79. AHRQ Publication No. 11-05149-EF-1. Rockville, MD: Agency for Healthcare Research and Quality; 2010

58. Pavey TG, Anokye N, Taylor AH, Trueman P, Moxham T, Fox KR, Hillsdon M Green C, Campbell JL, Foster C, et al. The clinical effectiveness and costeffectiveness of exercise referral schemes: a systematic review and economic evaluation. Health technol assess. 2011;15(44):i-xii. 1-254

59. Vierron E, Giraudeau B. Sample size calculation for multicenter randomized trial: taking the center effect into account. Contemp Clin Trials. 2007;28(4):451-8.

60. GM Fitzmaurice, Laird NM, and JH Ware: Applied longitudinal analysis; 2004

61. Sedgwick P. Cluster randomised controlled trials: sample size calculations. Bmj. 2013;346(f2839)

62. Pavey T, Taylor A, Hillsdon M, Fox K, Campbell J, Foster C, Moxham T, Mutrie $\mathrm{N}$, Searle J, Taylor R. Levels and predictors of exercise referral scheme uptake and adherence: a systematic review. J Epidemiol Community Health. 2012;66(8):737-44.

63. Gallegos-Carrillo K, Garcia-Pena C, Salmeron J, Salgado-de-Snyder VN, Vazquez-Cabrer G, Lobelo F. Exercise-referral scheme to promote physical activity among hypertensive patients: design of a cluster randomized trial in the primary health care units of Mexico's social security system. BMC Public Health. 2014;14:706.

64. Verbeke G, Molenberghs G, Thijs H, Lesaffre E, Kenward MG. Sensitivity analysis for nonrandom dropout: a local influence approach. Biometrics. 2001;57(1):7-14.

65. Bessa B, de Oliveira LV, Moraes C, Barboza J, Fouque D, Mafra D. Resistance training in hemodialysis patients: a review. Rehabil Nurs. 2015;40(2):111-26.
66. Konstantinidou E, Koukouvou G, Kouidi E, Deligiannis A, Tourkantonis A. Exercise training in patients with end-stage renal disease on hemodialysis: comparison of three rehabilitation programs. J Rehabil Med. 2002;34(1):40-5.

67. Heath $G$, Kolade $V$, Haynes JW. Exercise is medicine: a pilot study linking primary care with community physical activity support. Preventive Medicine Reports. 2015; In Press

68. Gallegos-Carrillo K, Garcia-Pena C, Salmeron J, Salgado-de-Snyder N, Lobelo F. Brief counseling and exercise referral scheme: a pragmatic trial in Mexico. Am J Prev Med. 2017;52(2):249-59.

\section{Ready to submit your research? Choose BMC and benefit from:}

- fast, convenient online submission

- thorough peer review by experienced researchers in your field

- rapid publication on acceptance

- support for research data, including large and complex data types

- gold Open Access which fosters wider collaboration and increased citations

- maximum visibility for your research: over $100 \mathrm{M}$ website views per year

At BMC, research is always in progress.

Learn more biomedcentral.com/submissions 